\title{
RASFX and DiFX: The Comparison of Geodetic VLBI Processing Results
}

\section{Voytsekh Ken}

Institute of Applied Astronomy of the Russian Academy of Sciences E-mail: ken@iaaras.ru

\section{Alexey Melnikov*}

Institute of Applied Astronomy of the Russian Academy of Sciences

E-mail: aemeiaaras.ru

In this work we present the comparison of group delays obtained by RASFX and DiFX correlators using different post-processing software. We found that the difference of the group delays from both correlators are mainly due to the different mathematical implementation of the correlation and post-processing algorithms. The data converter software have been developed which allows to use PIMA post-processing routine on RASFX correlator data instead of the native RASFX post-processing software WOPS. A series of intensive VLBI sessions were processed using this new approach.

14th European VLBI Network Symposium \& Users Meeting (EVN 2018)

8-11 October 2018

Granada, Spain

\footnotetext{
*Speaker.
} 


\section{Introduction}

GPU-based software correlator RASFX has been developed for geodetic processing of the VLBI observations in the IAA RAS in 2014 [1]. Since 2015, it participates in the processing of the observations with «Quasar» VLBI Network [2]. The RASFX software consists of the correlator processing software, ephemeris model software, and the wide-band observation post-processing system software (WOPS). RASFX utilizes the HPC cluster of the IAA RAS and outputs the calculated group delays in the NGS card files. DiFX [3] is installed on the same HPC cluster.

The series of intensive geodetic observations of two 13-m radio telescopes of the «Quasar» VLBI Network was carried on in September 7-26, 2018. There were 5 sessions in a day in dual band X/S setup on «Badary» — «Zelenchukskaya» baseline aimed to obtain UT1-UTC measure. Both 13-m radio telescope are equipped with the S/X/Ka Tri-Band receiver, the Broadband Acquisition System (BRAS), and the Data Transfer System (DTS). Observations were carried on in the following setup:

- One dual polarization S-band $512 \mathrm{MHz}$ bandwidth frequency channel,

- Two single polarization X-band $512 \mathrm{MHz}$ bandwidth channels,

- 2-bits sampling rate,

- 8 Gbps total data rate,

- 20 or 65 scans per session depending on scheduling strategy.

The data was transferred to the IAA Correlator Center in St.-Petersburg and was processed by two software correlators: RASFX and DiFX (version 2.4.1). The correlation was performed with the integration time of $\frac{1}{16} \mathrm{~s}$ for RASFX and $\frac{1}{2} \mathrm{~s}$ for DiFX, and spectral resolution of $250 \mathrm{kHz}$ for both correlators. Common geodetic observables are the group delays and group delay rates calculated from the auto- and cross-correlation spectra.

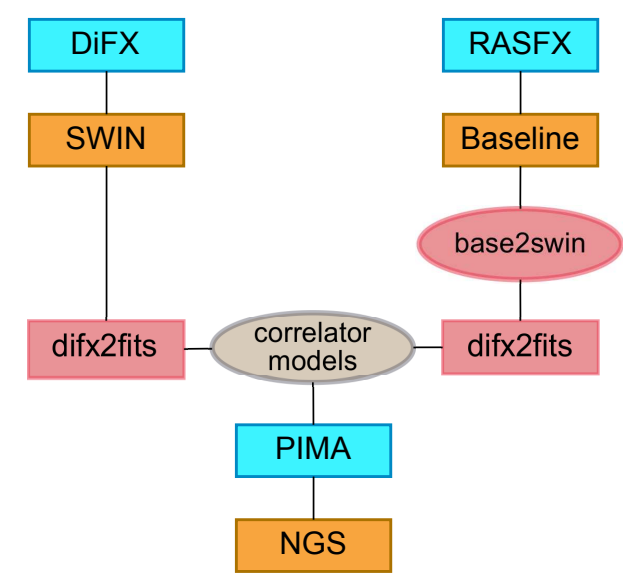

Figure 1: Data post-processing pipeline 


\section{Experiment description}

WOPS is the native post-processing software of the RASFX correlator which performs the calculation of the group delays, delay rates, and their formal errors, and signal-to-noise ratios. Then the CreateNGS program produces the NGS card files from this data.

The DiFX correlator outputs correlated data in the SWIN binary format which can be converted to the conventional FITS-IDI format using difx2fits program to use any software. Program difx 2 fits is a part of the DiFX distribution.

PIMA [4] is dedicated multi-purpose postprocessing software suite which inputs FITS-IDI files and makes the calculations of various parameters, including the group delays and rates, their formal errors, signal-to-noise ratios, and many others. The data output is in «geo VLBI data format» (GVF) or in plain text. GVF data can be processed with the $p$ Solve software and not related to this paper. For our purpose to compare the two correlators we have developed the pima2ngs data converter to produce the NGS card file from the plain text PIMA database files. Our standard post-processing pipeline is shown in Fig. 11.

Since PIMA requires FITS-IDI files we have developed several converter programs to use with the RASFX intermediate binary data in Baseline format. As shown in Fig. 1, the first step is to convert data from Baseline to SWIN format using base2swin converter. Program rasf 2 dif $x$ reads the correlator models from the RASFX control files and inputs these into DiFX correlator files. Then the difx2fits program is used to produce the FITS-IDI files. The final step is to make the NGS card files by using residual group delays and delay rates calculated by PIMA using CreateNGS program of RASFX. This step is necessary, since the a priori delays from PIMA differ from the RASFX correlator model.

\section{Data analysis}

Using the developed converter programs we have obtained NGS card files of 97 1-hour intensive session from both correlators and PIMA as the post-proceessing software (further in text 'DiFX+PIMA' and 'RASFX+PIMA'). Each X-band frequency channel was post-processed independentely, thus we have got two data series for each correlator: ' $\mathrm{X} 1$ ' for the high frequency channel of 8847.6 MHz center frequency and 'X2' for the low frequency channel (8335.6 MHz). Moreover, we obtain additional two series from standard RASFX processing routine which includes WOPS (further in text 'RASFX+WOPS').

Data series were sorted and compared by time marks and observed sources to ensure that only identical data point will be accounted, data points not presented in all these series were removed. The group delay in series 'DIFX+PIMA' was corrected for the clock offsets of the correlator, since PIMA do not add this value to the total group delay. Bias was removed from group delay values of each individual NGS card file. The differences of total group delays between the 'DIFX+PIMA' and 'RASFX+PIMA', and between the 'DIFX+PIMA' and 'RASFX+WOPS' were calculated. After a few iterations (5, exactly) of removing outliers by $3 \sigma$, we have obtained the final standard deviation values of group delay differences (see Table 1). The final reduced data of 'X1' frequency channel are presented in Fig. 2 and of 'X2' in Fig. 3, in these figures the red line with crosses 
corresponds to the difference between 'DIFX+PIMA' and 'RASFX+PIMA', and the blue line with circles - 'DIFX+PIMA' and 'RASFX+WOPS'.

\begin{tabular}{|l|l|l|}
\hline & $\sigma_{X 1}, \mathrm{ps}$ & $\sigma_{X 2}, \mathrm{ps}$ \\
\hline 'DIFX+PIMA' - 'RASFX+PIMA' & 17.1 & 16.5 \\
'DIFX+PIMA' - 'RASFX+WOPS' & 16.2 & 16.0 \\
\hline
\end{tabular}

Table 1: Standard deviations of the delay differences

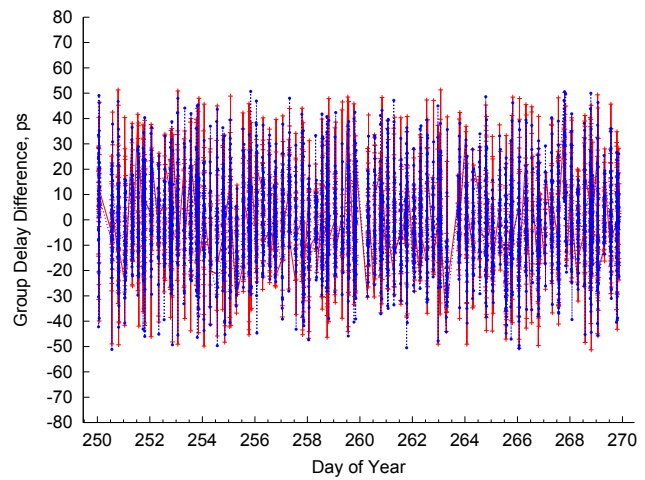

Figure 2: 'X1' frequency channel group delay difference

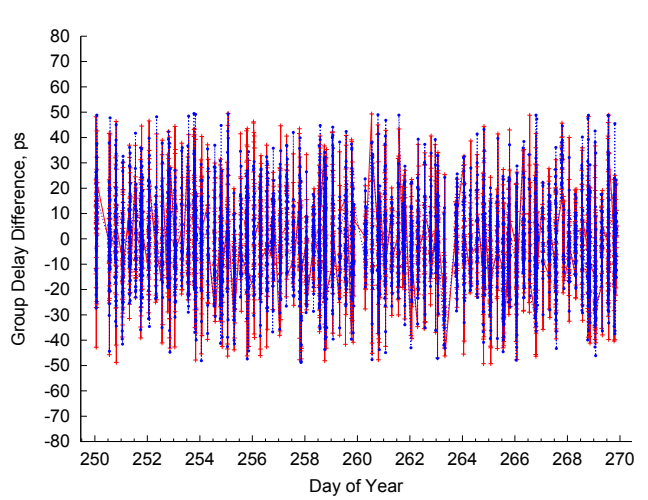

Figure 3: 'X2' frequency channel group delay difference

\section{Summary and conclusions}

The RASFX data converter software was developed and tested. Analysis of 97 1-hour sessions have shown that there is a systematic difference of $\approx 16.5$ ps between the RASFX and DiFX correlators. This systematic difference does not depend on the post-processing software and slightly differs for ' $\mathrm{X} 1$ ' and 'X2' frequency channels. While the calculated group delays from WOPS and PIMA software show good agreement and differ by 0.9 ps or less.

\section{Acknowledgements}

We are grateful to Igor Surkis for his assistance and discussion of the results.

\section{References}

[1] Surkis, I.F., Zimovsky, V.F., Ken, V.O. et al. Instrum Exp Tech (2018) 61: 772.

[2] A. Ipatov, D. Ivanov, G. Ilin et al. New Generation VLBI: Intraday UT1 Estimations // IVS 2016 General Meeting Proceedings "New Horizons with VGOS", Edited by Dirk Behrend, Karen D. Baver, and Kyla L. Armstrong NASA/CP-2016-219016, 106-110 (2016)

[3] Deller, A.T., Brisken, W.F., Phillips et al. (2011). DiFX-2: A More Flexible, Efficient, Robust, and Powerful Software Correlator. ASTRON SOC PAC. 123. 275-287.

[4] L. Petrov, Y.Y. Kovalev, E.B. Fomalont, D. Gordon. The VLBA Galactic Plane Survey VGaPS. Astron. J., 142, 35, 2011 